\title{
Semiperturbative construction for the quark-gluon vertex
}

\author{
Felipe J. Llanes-Estrada ${ }^{a}$, Christian S. Fischer ${ }^{b}$, Reinhard Alkofer ${ }^{c}$ \\ aUniversidad Complutense de Madrid, Depto. Física Teórica I. 28040 Madrid, Spain \\ ${ }^{b}$ IPPP, University of Durham, Durham DH1 3LE, U.K. \\ 'Institute for Theoretical Physics, University of Tübingen, D-72076 Tübingen, Germany
}

\begin{abstract}
We construct a model for the quark-gluon vertex of Landau gauge QCD. This is of twofold interest: on the one hand the quark-gluon interaction is at the heart of quark confinement, on the other hand it is a central element in hadron phenomenology based on QCD Greens functions. We employ the non-Abelian one-loop diagram in perturbation theory, which is of order $N_{c}$. As a novelty we replace the tree-level quark and gluon propagators in this diagram by their dressed counterparts solving the Dyson-Schwinger equations. The $N_{c}$-suppressed Abelian diagram is an order of magnitude smaller in various kinematics. We also study the effect of ghost dressing factors on the vertex obtaining a construction in good agreement with recent low-momentum lattice calculations.
\end{abstract}

\section{Construction of the vertex model}

The infrared suppression of the gluon 2-point function in QCD $[1,2]$ entails that the bare $q \bar{q} g$ vertex, usually employed in the rainbow truncation of the Dyson-Schwinger Equation (DSE), is insufficient to trigger dynamical chiral symmetry breaking. Therefore we expect an infrared enhancement in the quark and gluon vertex, as suggested also by its Slavnov-Taylor Identity (STI). A model vertex with a Ball-Chiu or Curtis-Pennington structure multiplied by enhancing ghost factors has been successfully employed in [3] in the quark DSE. For a short summary of these results see ref. [4].

In this work we report a diagrammatic construction based on the one-loop perturbative QCD corrections to the bare vertex [5].

There are two relevant diagrams, to which we refer as Abelian and non-Abelian due to the vertex attached to the gluon (as is customary). In both diagrams we substitute the quark and gluon propagators by their dressed counterparts solving the DSE's [3]. These are added to the bare vertex $Z_{1 F} \gamma_{\mu}$ and the renormalization constant is fixed by imposing that the $\gamma_{\mu}$ component is unity at a renormalization point of $2 \mathrm{GeV}$.

The non-Abelian diagram, depicted in figure 1 dominates over the Abelian one nominally by a

0920-5632/\$ - see front matter C 2005 Publish by Elsevier B.V. doi: $10.1016 /$ j.nuclphysbps.2005.08.008 factor $N_{c}^{2}$. We have checked that 1) this dominance by an order of magnitude remains after loop integration in the vertex for various kinematics and 2) the impact of the Abelian vertex on the quark DSE is smaller by the same fartor by performing a kinematic average with the kernel of the DSE as weighting function. Therefore to a precision of $10 \%$ or even better one can ignore the Abelian diagram.

\section{Numerical results}

The vertex is projected into the tensor basis from appendix $A$ in [6]. The loop integral is calculated numerically in four dimensions with a standard Gauss-Legendre grid. In one computer code we perform the spin sums numerically, in an alternative calculation we employ a form code to reduce the kernel analytically to relatively complex but tractable scalar integrals.

If the internal $q \bar{q} g$ vertices are taken as bare $Z_{1 F} \gamma_{\mu}$ then the construction, that qualitatively has the right behaviour, is not strong enough to reproduce lattice data and trigger chiral symmetry breaking in the quark DSE. This, as commented above, is expected and can be remedied by enhancing the internal vertices by a ghost dressing factor. In tigure 2 we compare the resulting $q \bar{q} g$ vertex with lattice data at the so 


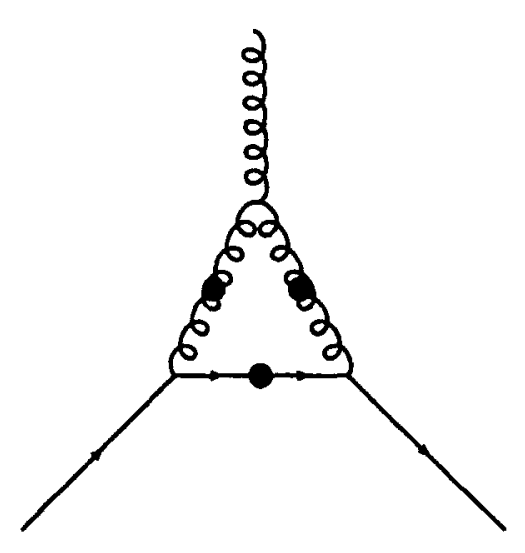

Figure 1. The non-Abelian one-loop correction to the quark and gluon vertex is the basis of our nonperturbative model. To this end we replace internal propagators by their dressed counterparts and enhance the internal vertices with one ghost dressing function each reflecting the STI.

called "asymmetric point" characterized by $p_{g}=$ $0, p_{1}=p_{2}$ where the gluon momentum vanishes, $p_{1}$ flows into the vertex and $p_{2}$ exits. This comparison is successful for the leading Dirac amplitude $\lambda_{1}$ and the scalar amplitude, $\lambda_{3}$. The amplitude $4 p^{2} \lambda_{2}$ vanishes in our model at low momenta, whereas the lattice data (with large errors) seem to approach a constant value implying a divergence of $\lambda_{2}$.

We now investigate a more interesting section of kinematic space, that we denote "totally asymmetric" point, characterized by the relations $p_{2}=2 p_{1}$ and $p_{g}=3 p_{1}$ between the moduli of the momenta. This point is interesting because the tensor basis used is non-singular and all twelve different Dirac amplitudes $\lambda_{1-4}, \tau_{1-8}$ contribute to the vertex. We plot the four leading structures in figure 3.

The other eight Dirac amplitudes are increasingly smaller, down to two orders of magnitude below the plotted ones. Thus there is a rich hyrarchy of Dirac amplitudes that can help in model building.

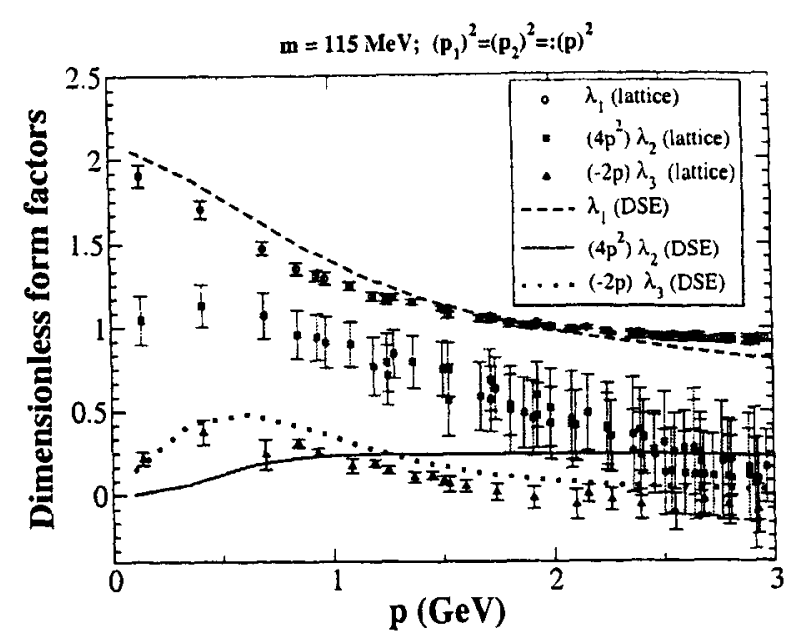

Figure 2. Comparison of our results with lattice data in the particular kinematic section $p_{1}=p_{2}$. $m_{q}(2 \mathrm{GeV})$ is set to $115 \mathrm{MeV}$ but similar results are obtained at the other lattice data set available at $60 \mathrm{MeV}$.

\section{Mass dependence and Chiral Symmetry Breaking}

Once we are in possession of a construction that successfully compares to lattice data, we employ it to perform a study of the mass dependence of the vertex. First note the Abelian one-loop diagram contains two quark propagators, and is therefore suppressed as $1 / M_{q}^{2}$ in the heavy quark limit, whereas the non-Abelian diagram will damp as $1 / M_{q}$. Therefore future calculations relating observables in the charmonium and bottomonium systems (see [8] for discussion) will be sensitive to what class of diagrams enters the vertex model.

An interesting observation is that for the range of quark masses considered in the lattice calculations, the $\lambda_{3}$ Dirac amplitude has a maximum. If the current quark mass is further increased, the intermediate quark propagator suppresses the vertex loop. Conversely, approaching the chiral limit, the quark mass function takes its minimum value dictated by chiral symmetry breaking alone 


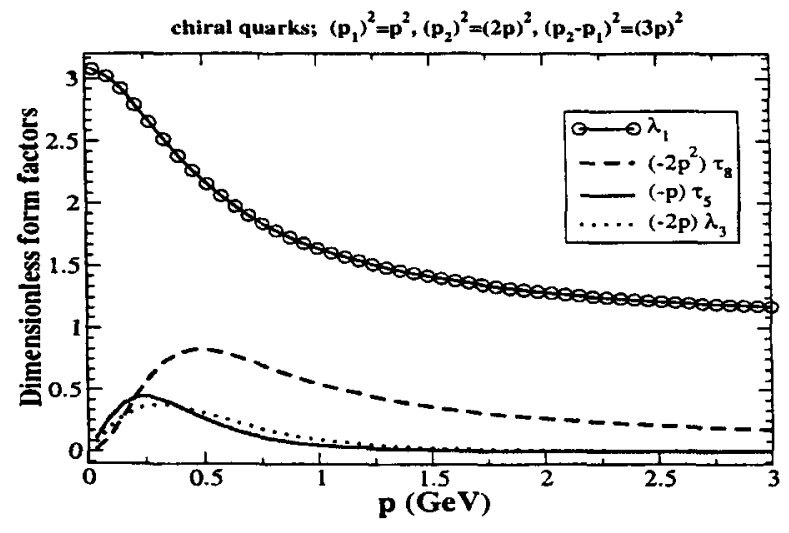

Figure 3. Leading tensor structures in the kinematic section given by $p_{1}:=p, p_{2}=2 p, p_{g}=3 p$ in our model construction.

and the scalar part of the loop construction also has a minimum (slight corrections to this result are expected after self-consistently solving a vertex equation).

As for the leading $\lambda_{1}$ amplitude, we plot in figure 4 the quotient $\lambda_{1} / A$, where deviations from unity signal departures from the Abelian WardTakahashi identity.

\section{Outlook}

Results similar to ours have been independently obtained [9] in a different scheme. This exploits the STI for the three-gluon vertex to model it. Since this amounts to the resummation of a totally different class of diagrams we would need better lattice data to distinguish both models. Our construction is of course valid (within approximations) for all possible kinematics and not just when the gluon momentum vanishes. On the positive side, both works concur in predicting $p^{2} \lambda_{2}\left(p^{2}\right)$ to vanish as $p \rightarrow 0$. We should note that the existing lattice data, with large error bands, suggests instead this limit is finite, implying a divergence in the vertex, in the Dirac amplitude $\lambda_{2}$.

When multiplied by appropriate powers of $p$ the resulting dimensionless Dirac amplitudes of

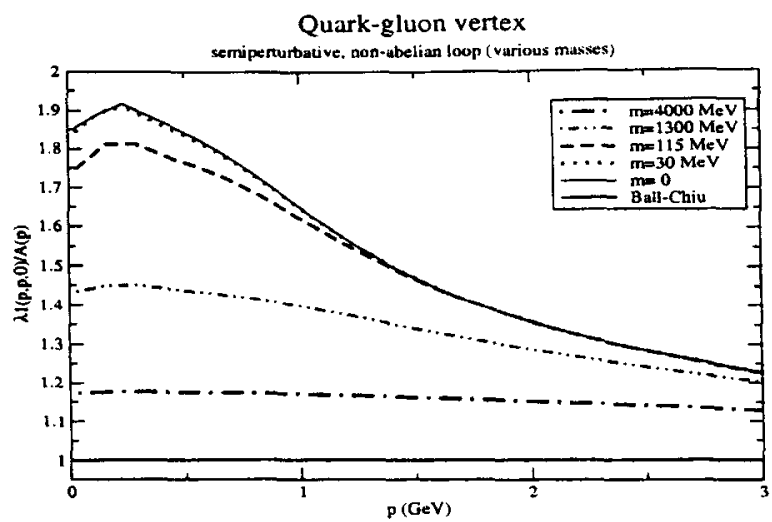

Figure 4. Mass dependence of the quotient $\lambda_{1} / A$ that equals 1 in an Abelian theory (as incorporated in the Ball-Chiu construction).

our vertex construction vanish as $p \rightarrow 0$ except the leading structure $\lambda_{1}$ that takes a finite value.

Whether a divergence can arise as a consequence of the feedback of the obtained vertex model on the loop construction itself (implying a self-consistent solution is needed) or as a consequence of the backreaction on the quark SDE is a topic under current scrutiny. The construction of a quark scattering kernel based in our model vertex and consistent with chiral symmetry is now straight-forward.

\section{Questions from the audience.}

- How are Euclidean space singularities in the two-point functions mapped to Minkowski space after analytical continuation?

This is a nonperturbative problem and we lack a full answer at this stage. One has obtained some understanding of the analytical structure of the two-point functions in a recent work [10]. Also Hamiltonian calculations in Coulomb gauge, not in Euclidean space, provide clear evidence for the picture of ghost enhancement and gluon suppression at low momentum [11]. 
- The running $\alpha_{s}$ you are employing seems to be somewhat too high in the middlemomentum range. What is the impact of this in the results reported?

This study does not aim at precisely pinpointing details of the propagators and vertex functions, but to obtain insight into their qualitative features and structure, and identify possible divergences that may otherwise escape lattice calculations. In this respect, the impact of this few-percent deviation can be ignored.

- Why is the dressing of the three-gluon vertex omitted?

As can be observed in figure 5 , all dressing of the triple gluon vertex can be absorbed in the quark-gluon scattering kernel. Our model based on the one-loop correction to the qqg vertex can be also viewed as an approximation to this kernel. In this exact equation [12] we are neglecting completely the two last terms, involving the ghostquark scattering kernel and the three-gluonquark scattering kernels, as their skeleton exDansion starts at two loops.

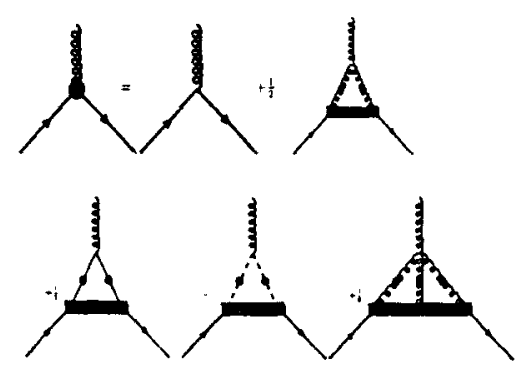

Figure 5. Exact vertex DSE equation.

We thank M. Bhagwat, C. D. Roberts and P. Tandy for valuable discussions. The lattice data in figure 2 are a courtesy of the authors of [7]. F. L. E. thanks the hospitality of the Tübingen Institute as well as a DAAD stipendium and Univ. Complutense travel grant. Partial support from grants FPA 2000-0956, BFM 2002-01003 (MCYT, Spain), Al 279/3-4, Fi 970/2-1, and GRK683 (DFG, Germany).

\section{REFERENCES}

1. R. Alkofer and L. von Smekal, Phys. Rept. 353 (2001) 281 [arXiv:hep-ph/0007355].

2. P. O. Bowman et al., arXiv:hep-lat/0402032.

3. C. S. Fischer and R. Alkofer, Phys. Rev. D 67 (2003) 094020 [arXiv:hep-ph/0301094].

4. C. S. Fischer, F. Llanes-Estrada and R. Alkofer, arXiv:hep-ph/0407294.

5. A. I. Davydychev, P. Osland and L. Saks, Phys. Rev. D 63 (2001) 014022

6. J. Skullerud and A. Kizilersu, JHEP 0209 (2002) 013 [arXiv:hep-ph/0205318].

7. J. I. Skullerud, P. O. Bowman, A. Kizilersu, D. B. Leinweber and A. G. Williams, JHEP 0304 (2003) 047 [arXiv:hep-ph/0303176].

8. M. S. Bhagwat, A. Holl, A. Krassnigg, C. D. Roberts and P. C. Tandy, arXiv:nuclth/0403012.

9. M. S. Bhagwat and P. C. Tandy, arXiv:hepph/0407163.

10. R. Alkofer, W. Detmold, C. S. Fischer and P. Maris, Phys. Rev. D 70 (2004) [arXiv:hep$\mathrm{ph} / 0309077$ ].

11. A. P. Szczepaniak and E. S. Swanson, Phys. Rev. D 65 (2002) 025012 [arXiv:hep$\mathrm{ph} / 0107078] ; \quad$ D. Zwanziger, arXiv:hepph/0312254; C. Feuchter and H. Reinhardt, arXiv:hep-th/0402106; id. work in preparation.

12. W. J. Marciano and H. Pagels, Phys. Rept. 36 (1978) 137; E. Eichten and F. Feinberg, Phys. Rev. D 10 (1974) 3254. 